\title{
SCIDic
}

International Journal of Translation \& Community Medicine (IJTCM)

ISSN: $2333-8385$

\section{Translation Mode of TCM Classic Nan Jing in the United States}

Feng Zhijiao*

South West Jiaotong University, Lanzhou City University, China.

\section{Abstract}

Since the outbreak of new coronavirus COVID-19 in 2019 and its evolution into a global disaster, traditional Chinese medicine (TCM) helps thousands of people get out of the catastrophe. How to get access to TCM knowledge highlights the mission of translation. Nan Jing, one of four medical classics in China, has played a pivotal role in the medical development of TCM. On the basis of the five communication elements of Lasswell, the study takes Nan Jing's English translation in the United States as the research object, analyzes thetranslation mode of Nan Jing from the perspectives of translation subjects, translation contents, translation channels, target readers and translation effects so as to provide some references for the English translation of TCM classics in western world and subsequently improve people's awareness of disease treatment and prevention.

Keywords: Nan Jing; Translation Subjects; Translation Contents; Translation Channels; Target Readers; Translation Effects.

\section{Introduction}

Nan Jing, originally named Yellow Emperor's Eighty-one Difficulties, is one of the four classic books of traditional Chinese medicine (TCM). The accomplishment of this classic was before the Eastern Han Dynasty without a clear record of its author, while scholars has a disposition of Bianque. Nan Jing is of question and answer writing format to discuss the eighty-one difficulties of TCM. As a supplimentry work of HuangdiNeijing (The Yellow Emperor's Canon of Internal Medicine), Nan Jing is the essence of TCM, short in length and condensed in content. The early western translation of Nan Jing was firstly published by Hubotter F, medical history specialist, University of Berlin, in offprint in 1929. While the first English version of Nan Jing was translated and published by German Professor Paul U. Unschuld in 1986, and the first English version of Nan Jing in China was published by Professor Li Zhaoguo in World Publishing Corporation in 2008. Compared with the research devotion of HuangdiNeijing, Nan Jing has received less attention both in China and abroad, and its research value needs to be further explored.

Medio-translatology researches on translation from the perspective of comparative literature, mostly about translation literature and literary translation [8]. Translation is a cross-cultural commu- nication activity and literary translation is a cultural communication behavior. The proposition of five communication elements by famous American sociologist, Lasswell, are namely "Who? What to say? By what means? To whom? What effect?" [3]. The above five elements correspondingly conform to the translation subject, translation content, translation channel, readers and translation effect inmedio-translatology. This research tends to analyze translation mode of Nan Jing from the above elements with an expectancy to provide some references for the overseas dissemination of TCM.

\section{Sinologists as Main Translation Subjects}

The translation subject involves "who will translate?", including the translator, the sponsor and even the author. Translation subject determines the translation content and strategy. Thence, the popularity and recognition of translation subject affect the dissemination effect of the original text [10]. In 1986, Paul U. Unschuld, a famous historian of TCM, a sinologist, and a translator of Chinese medical classics,the director of the Institute of Medical History of University of Munich, Germany, published the first English version of NAN CHING-The Classic of Difficult Issues at the University of California. In 1991, Paul U. Unschuld cooperated with Zheng Jinsheng, an expert of TCM literature at

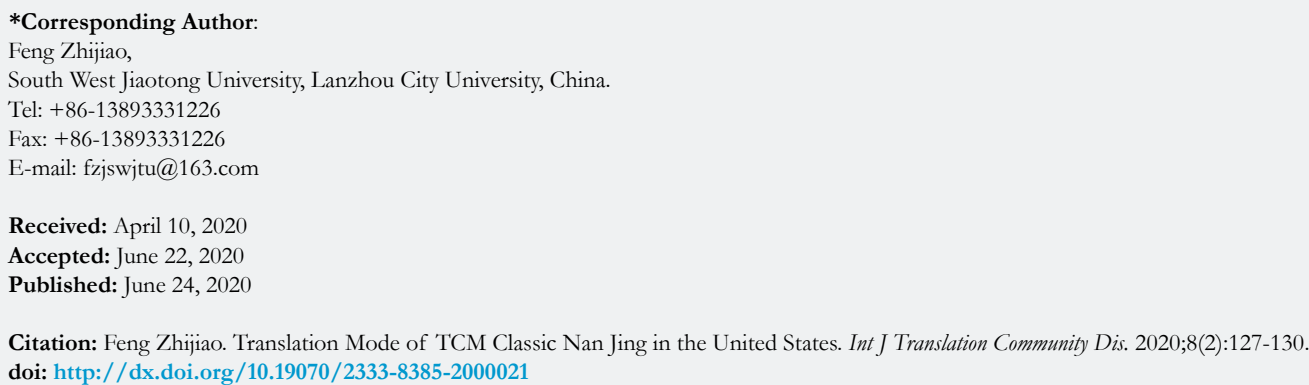

Copyright: Feng Zhijiao 2020 . This is an open-access article distributed under the terms of the Creative Commons Attribution License, which permits unrestricted use, distribution and reproduction in any medium, provided the original author and source are credited. 
the Chinese Academy of Chinese Medical Sciences, and Hermann Tessenow, a well-known scholar in the United States, and translated The Yellow Emperor's Nei Jing Su Wen, till 2011, the accomplishment got published at University of California Press. In addition to the translation of TCM classics, Paul U. Unschuld has authored a series of TCM monographs, such as Medicine in China: A History of Ideas in 1985, Medicine in China: A History of Pharmaceutics in 1986, What Is Medicine?: Western and Eastern Approaches to Healing in 2009, etc. His achievements in spreading TCM culture won Paul U. Unschuld the "Special Book Award of China" in 2017.

Bob Flaws, born in New Jersey in 1946, won popularity as a sinologist, translator and lecturer of TCM in the United States, and one of the founders of the Blue Poppy Enterprise in American TCM industry.In 1977, Bob Flaws came into contact with TCM from Dr. Eric Tao in Denver, who traveled to Taiwan because of his Kuomintang identity and then to the United States. Later, Flaws went to Shanghai College of TCM to study acupuncture, Chinese herbal medicine and massage. In 1999, Flaws published The Classic of Difficulties: A Translation of Nan Jing in Blue Poppy Publishing House. Prior to this, his cooperation with Yang Shouzhong, a Chinese medical expert, contributed to the English translation of Master Hua's Classic of The Central Viscera in 1993. Besides, Bob Flaw published TCM monographs, such as The Tao of Healthy Eating: Dietary Wisdom According to Traditional Chinese Medicine in 1998, The Secret of Chinese Pulse Diagnosis in1999 and Chinese Medical Psychiatry in 2010, etc and won great popularity. It can be found that Bob Flaws has played an important role in the spreading of TCM culture in the United States.

Dr. Dong Hua is currently the president of Sharel Zedek Medical Center and a professor at the Atlantic Oriental Medical Institute in Fort Lauderdale, Florida. From 1996 to 2005, Dr. Dong Hua pioneered the Chinese therapy in Israel and lectured massage to hundreds of doctors and students. In 2007, he established SharelZedek Medical Center in Fort Lauderdale, Florida and dedicated to research on acupuncture and anesthesia. Dr. Dong Hua has won wide acclaim with his clinical practice in TCM and is widely known as "golden hands" and "magic hands".

\section{Translation Contents}

André Lefevre pointed out that translation must be dominated by the ideology and literary views of the translator or the authority, and translated version can not truly reflect the original text. Lefevre believes that translation, restricted by ideology, patronage and poetic concepts, is a kind of re-creation [6]. The translation content is namely "what to translate". It is greatly influenced by ideology, social trend, and translator's subjective cognition. All the prior factors make translators balance the cultural characteristics of the source language, preferences of the readers, and the social and cultural norms, and consequently adopt the strategies to add, delete, edit, and rewrite the original text for the realistic purposes. In 1980s, Paul U. Unschuld found a large number of western TCM works were not in line with the authenticity of TCM, and were mostly "fabricated". Therefore, he determined to engage in the translation of ancient Chinese medical classics and clarify the root of TCM. Paul U. Unschuld advocates that the translation of TCM classics should strictly follow the principles of linguistics, accurately explore the formation process of TCM terms, probe into the roots of images and metaphors in the original text, select appropriate vocabulary with the assistance of annotations so as to truly and completely representthe TCM classics [10].The English version of Nan Jing by Paul U. Unschuld simultaneously presents all the original texts so that readers with Chinese knowledge background can make a comparison on their own. In addition, a large number of important annotations of Nan Jing from China and Japan for centuries are for the first time quoted in his version. In 2016, Paul U. Unschuld revised the previous edition of Nan Jing from the perspectives of philology, methodology, and sinology to highlight the philosophical balance of five-elements and yin-yang.

On the whole, Bob Flaws' version is also a full translation. As he mentioned in the preface, the main purpose of his version is to introduceNan Jing to English readers. In order to meet the public's reading taste, Flaws adopts relatively flexible processing strategies to improve the acceptability [1]. For the contents that must be added, Flaws supplements them with parentheses to enhance readability. Example as below: the third difficulty said “关 之前者, 阳之动也, 脉当见九分而浮。过者, 法曰太过; 减者, 法曰不及。” Flaws' version is as follows: In front of the bar is where yang stirs. The pulse seen in those nine fen is floating. [If extending] beyond [these nine fen], this is called greatly excessive. If vacuous, this is called not reaching.

The third difficulty mainly discusses the covering and overflowing pulse, which is a phenomenon in which yin and yang of the human body are uncoordinated, and the pulse appears too much or less. Here is a term “关”, namely, "bar”. In his version, Flaws supplements the indispensable information of "bar", he adds "If extending" and "these nine fen" in the parentheses to help readers recognize the special position of this "bar".

Dr. Dong Hua's version, also a full translation, presents the key information of each difficulty in a concise format, which conversely exposes some problems of his translation, such as a shortage of accurate interpretation of TCM terms, even mistranslation of key terms. The original ninth difficulty said,“曰: 何以别知脏腑之 病耶? 然: 数者腑也, 迟者脏也。数则为热, 迟则为寒。 诸阳为热, 诸阴为寒。故以别知脏腑之病也。Dong Hua's corresponding translation is as below: "How can we diagnosis if it is fu or zang disease according to the pulse? Answer: Rapid pulse indicates fu disease. Slow indicates zang disease. Rapid pulse is most likely seen in heat symptoms. Slow pulse is most likely seen in cold symptoms. Fu belong to the Yang. Therefore, fu disease manifests heat symptoms. Zang belong to Yin. Zang disease manifests cold symptoms. So we can feel the pulse is rapid and slow to diagnosis if it is zang or fu disease."

"Comments: Rapid pulse, which is yang pulse, indicates excess or heat yang syndromes in Fu organs; slow pulse, which is ying pulse, indicates cold or deficiency syndromes in Zang organs because Fu organs are Yang Organs and Zang Organs are ying organs."

Each difficulty of Dr. Dong Hua's version includes two parts, translation and comment. His translation is a general translation of the original text and the comment supplements the relevant information of key terms. However, from the perspective of the overall format, his version is in scarcity of explanations and origin studies of related TCM terms, such as "yin" and "yang", "zang" and " $f u$ ", and his version even arise mistranslations of core terms “阴” into "ying” and inconsistency of letter cases. It can be seen that Dong Hua's version can be used as a popular book for read- 
ers to capture a global view of Nan Jing. However, insufficiency research on the original text and inadequate verification of key terms make it unsuitable for professional readers to understand cultural terms and essence of TCM.

\section{Well-known American Publishing Houses as Translation Channels}

Translation channel, that is, the way in which the original text and culture are disseminated, including publishing houses, radio stations, and online media. The choice of the translation channel is closely related to the dissemination effect of translated works. Choosing a publishing house with widespread impactbrings positive result for the overseas dissemination of TCM culture. It can not only enlarge the number of readers of translated works, but also enhance the cultural influence of translated works in Western society. Paul U. Unschuld's translation of Nan Jing was published by University of California, Bob Flaws' version was by Blue Poppy Press, and Dong Hua's was by Create Space Independent Publishing Platform respectively.

Founded in 1893, the University of California Press is one of the six largest university presses in the United States. Initially, it was a small publishing organization dedicated to publishing books for the staff of University of California. Since its springing up as a world-class university press in 1950s, it dedicates to scientific research, professional education and public services. The New York Times commented: "Asian studies, literary criticism, and sociological studies are the strengths of the University of California Press [5].” Its adherence of rigorous academic norms helps publications win profound academic and social impact, and the same with Nan Jing. Since its publication, related researches successively emerge on important medical journals both in China and abroad, such as Pacific Journal of Oriental Medicine, American Acupuncturist, Oriental Medicine, California Journal of Oriental Medicine. All the researches above have expanded the academic influence of Nan Jing overseas.

Blue Poppy Enterprise Co., Ltd. was founded in 1982 by Bob Flaws and Honora Wolfe, the pioneers of TCM in the United States. This Enterprise is dedicated to the publication of Chinese medical books, promotion of TCM acupuncture, Chinese herbal medicine products and public health as well as prevention of diseases.It becomes an important booster of Chinese medical products in the United States. Blue Poppy Publishing House was initially founded to solve the problem of the extreme lack of English TCM books in the early rise of the Chinese medical industry in the United States. Up to now, more than 100 kinds of TCM books have been published, covering the English versions of TCM classics, study of acupuncture, pediatrics and herbal medicine.

CreateSpace Independent Publishing Platform grew out of a small little known publishing house established in 2000 in Scotts Valley, California, USA. The publishing house cooperated with a movie online platform, namely Custom Flix Labs to provide scholars with online publishing services. It was acquired by Amazon in 2005 and subsequently renamed CreateSpace. Generally, books on CreateSpace are only published through Amazon or are available for reading through the Kindle format. Nowadays, Amazon.com has become the world's largest book sales website, so books on it can be delivered to all parts of the world and favored by readers worldwide.

\section{Multi-level Target Readers}

The target readers are the reception and feedback group of a translation [9]. Paul U. Unschuld's translation is in Chinese-English formattogether with researches on the original Chinese text and Japanese annotations. In general, the length of Paul U. Unschuld's notes and commentaries is much longer than translation itself, which provides sufficient academic references to Nan Jing. It can be found from the format that this version offers readers with a Chinese knowledge background to compare the original text and translation. It also supplies comprehensive academic references for readers to understand the relevant medical terms. In this view, Paul U. Unschuld's translations of other Chinese medicine classics, for instance, Huang Di's Inner Classic in 2011, have also retained the similar format. Paul U. Unschuld's format provides a content mode for TCM in the West, ie. original text, translation, comments and notes [2].

Bob Flaws has a clear account of hisreaders in his preface, including "any one who is interested in Chinese pulse diagnosis, the Chinese medicine of systematic correspondences in general and five phase theory in particular and Chinese acupuncture in particular [1]." Flaws also mentioned that his version is intended to retain the concise and lean style of Nan Jing, for those information that must be added, Flaws adopts parentheses to add annotation. From the target readers and format of Flaws' version, it can be found that the his version is mainly aimed at readers who are interested in TCM, rather than TCM professionals. Therefore, his version is only a popular reading [7].

Dong Hua is a Chinese medicine practitioner. His translation of Nan Jing is intended to provide readers with clinical guidance in TCM. His version has omitted many medical terms and is in dearth of interpretation of medical theory. Therefore, his readers are required to have a certain theoretical foundation of TCM, such as students in TCM majors and practitioners of TCM or readers who only want to capture a rough view of Nan Jing, so the reader group is slightly confined.

\section{Mixed Translation Effects}

The effect of translation is the degree to which readers accept the translation in the target culture, and it is also an important criterion for measuring the success of the entire translation process. Amazon website (Amazon.com) is the world's largest book sales website and has formed the largest online book review database. Paul U. Unschuld's translation has been widely acclaimed on the Amazon platform and is regarded as "an authoritative translation, a remarkable accomplishment". Readers believe that Paul U. Unschuld's version brings readers the most authentic look of TCM with detailed annotations, historical context of the original text and professional review on the essence of TCM culture. Meanwhile, Paul U. Unschuld's version supplies an angle to reexamine TCM theory and an exploratory path to engage in TCM practice. Despite praises, there are different voices. Flaws accounts that Paul U. Unschuld's translation is too redundant and his non-native English speaker identity make the authenticity of Nan Jing been questioned [1]. Bob Flaws' version is a general translation, which 
leads to the distortion and omission and even rewriting of some information in the original text, incompatible with the translation norms of cultural classics. Therefore, many readers believe that there is an insufficient information problem in his version. Dong Hua is a TCM practitioner, so his application of concise and capable words to clarify the obscure TCM theory in the original text brings about many linguistic errors and insufficient elaboration of the text. Therefore, its social influence is relatively weak for its low readability.

After the emergence of many English versions, the first English translation of Nan Jing in China was produced by Professor Li Zhaoguo and published in World Publishing Corporation in 2008, ending the situation of Nan Jing translation only by foreigners. Besides, Canadian Chinese LüCongming published A Complete Translation of Nei-Jing and Nan-Jing, which expanded the influence of Nan Jing in Canada.It can be seen that although there are multiple translations of Nan Jing, new full and abridged translations are still emerging, and its value in the medical field has drawn the attention and recognition of many scholars, contributing to the construction of a unique discourse system for TCM culture in the context of globalization.

Successive translations of Nan Jing in the United States have allowed English readers to have a deeper comprehension of the academic value of it, and also promoted the spread of TCM culture in the world. American TCM expert Joseph Adamsdiscussed the diagnosis of pulse and acupuncture in Nan Jing in the American Acupuncturist magazine in 2006, and published the application of double-deep pulse diagnosis principle in Nan Jing to the medical evaluation of yin-yang organs in the California Journal of Oriental Medicine in 2010, with specific citation of the 16th and 69th difficulties of Paul U. Schuld's version and the $61^{\text {st }}$ difficulty of Bob Flaws' version.Adams Joseph believes that Nan Jing is closely related to present society, and encourages the application of Nan Jing to clinical practice for justifiable evaluations [4]. Professor Rosenberg Z'EV in Oriental Medical College of the United States also published TCM teachings on pulse in Nan Jing in Oriental Medicine in 2018, and published the book Ripples in the FlowReflections on Vessel Dynamicsin theNan Jing in 2009, focusing on the thoughts and applications of pulse theory.

\section{Conclusion}

As a whole, the process of Nan Jing translation in the United States is closely related to the five elements of medio-translatology. The knowledge background and social identity of the translation subjects, the reading preferences and aesthetic interests, and mainstream tendencies of target culture have prompted translators to select the original texts, control translation content, and optimize translation strategies to achieve better translation effects of Nan Jing in the United States. Judging from the overall situation of Nan Jing translation, it can be inferred that by making appropriate judgment and control of the translation subjects, content, strategies and readers can TCM classics produce good effects overseas.

All in all, the author claims that under the "Going out" strategy of Chinese culture, a proper TCM translation mode is in great need. First of all, a translation mode of cooperation between Chinese and foreign translators is good for the preservation and presentation of TCM culture, for instance, the cooperation between Chinese medical experts, translators of Chinese medical classics and Chinese sinologists with a profound study on Chinese medicine. Secondly, the translation of TCM should satisfy the reading favors and aesthetic interests of the target readers. Thirdly, in terms of TCM culture spreading, well-known foreign publishing institutions are the better choices to expand the effect of TCM classics. Last but not least, after the publication of English versions, the translation effect should be tracked timely for further references to the later translation of TCM classics and expansion of TCM influence.

\section{References}

[1]. Bob Flaws. The Classic of Difficulties: A Translation of the Nan Jing. Blue Poppy Press, 1999.

[2]. Unschuld PU. Nan Jing: The Classic of Difficult Issues. Univ of California Press; 2016 Jul 19.

[3]. Lasswell HD. The structure and function of communication in society. The communication of ideas. 1948;37(1):136-9.

[4]. Adams J. Bringing the Nan Jing to life pulse taking and diagnosis from the classic of difficulties. Am Acupunct. 2006;35:25-7.

[5]. Kleinfield NR. How Six Press On[N]. New York Times. 1979; 10-21.

[6]. Lefevere, Andre.Rewriting and the Manipulation of Literary Fame. Routledge. 1992.

[7]. National Administration of Traditional Chinese Medicine. Guidelines for TCM Culture Construction in Traditional Chinese Medicine Hospitals. China Traditional Chinese Medicine Journal, 2009.

[8]. Paul U Unschuld.Nan Jing: The Classic of Difficult Issues (second edition). University of California Press, 2016jul 19.

[9]. XieTianzhen. Translatology. Yilin House. 2013.

[10]. Yin Li. Study on the Overseas Translation Mode of HuangdiNeijing and the "Go out" of TCM Culture [J]. Journal of PLA University of Foreign Languages. 2017; 40(6): 53-61.

[11]. Zheng Jinsheng. The Road of Chinese Medical Research by Professor Paul UUnschuld. Chinese Journal of History of Science and Technology. 2013; 1: 1-17. 